\title{
Enforcing stability constraints in Set-membership identification of linear dynamic systems
}

\author{
Vito Cerone $^{\S}$, Dario Piga, Diego Regruto \\ Dipartimento di Automatica e Informatica, Politecnico di Torino, Corso Duca degli Abruzzi 24, 10129 Torino, Italy
}

\begin{abstract}
In this paper we consider the identification of linear systems, a priori known to be stable, from input-output data corrupted by bounded noise. By taking explicitly into account a priori information on system stability, a formal definition of the feasible parameter set for stable linear system is provided. On the basis of a detailed analysis of the geometrical structure of the feasible set, convex relaxation techniques are presented to solve nonconvex optimization problems arising in the computation of parameter uncertainty intervals. Properties of the computed relaxed bounds are discussed. A simulated example is presented to show the effectiveness of the proposed technique.
\end{abstract}

Key words: Error-in-variables, linear systems, LMI relaxation, Set-membership identification, stability constraints.

\section{Introduction}

Any system identification procedure involves three basic ingredients: a set of input-output measurements, a set of candidate models and an identification method, which can roughly be described as a rule to select a model among the candidate ones, on the basis of the measured data and a proper model quality assessment criterion. The choice of the set of candidate models, sometimes called model structure, is the most critical step since it strongly relies on the available a priori information: practical experience, physical insights and engineering intuitions play here a crucial role. Restricting our attention to the case of linear time invariant (LTI) systems, bounded input bounded output (BIBO) stability is perhaps the most common assumption when open-loop identification procedures are of interest. In fact, openloop experiments cannot be performed in practice when this hypothesis is not satisfied. Although the system to be identified is often known to be stable, most of the proposed techniques do not exploit such a priori information in the definition of the assumed model structure since formal inclusion of stability constraints makes the estimation problem difficult to be solved. As a result, the identification procedure may give rise to inaccurate and

\footnotetext{
$\star \S$ Corresponding author V. Cerone. Tel. +39-(0)115647064 Fax +39-(0)11-5647064

Email addresses: vito. cerone@polito.it (Vito Cerone), dario.piga@polito.it (Dario Piga), diego.regruto@polito.it (Diego Regruto).
}

possibly unstable models, especially in the presence of shortage of data, modeling error and measurement noise. Identification of input-output stable linear dynamic systems described by difference equations is considered in (Söderström and Stoica, 1981) where, through a simple counterexample, it is shown that the least squares (LS) method may lead to unstable models when certain conditions in terms of signal-to-noise ratio are satisfied. Only few contributions are available in the literature that address the problem of how taking into account a priori information on system stability. A sufficient condition to ensure stability of models obtained by LS identification is provided in (Regalia and Stoica, 1995) where the input signal is constrained to be an autoregressive process of a given degree. Tugnait and Tontiruttananon in (Tugnait and Tontiruttananon, 1998) provide a frequency domain solution to LS identification of a stable system in the presence of undermodeling. Their approach can be applied when the input signal is a zero-mean stationary process with sufficiently high persistency of excitation order. A stable output error identification scheme is presented in (Janakiraman and Bhattacharyya, 1999) for the case of all-pole systems and periodic excitation signals, while a procedure to include a priori information on BIBO stability in the context of the kernel-based nonparametric identification is discussed in (Pillonetto and DeNicolao, 2010). As far as subspace identification is concerned, different approaches have been proposed to enforce stability (Chui and Maciejowski, 1996; Gestel et al., 2001; Lacy and Bernstein, 2003).

In this work, we consider the identification of single input 
single output (SISO) discrete-time linear systems that are a priori known to be stable. The aim of the paper is to show how to enforce stability in the identification of the system parameters. In particular, we focus our work on the computation of parameter bounds, taking explicitly into account a priori information on system stability, in the set-membership error-in-variable (EIV) framework, that is identification of dynamical systems when both input and output signals are corrupted by bounded noise. The reader can find further details on the set-membership approach in a number of survey papers (see, e.g., (Milanese and Vicino, 1991; Walter and PietLahanier, 1990)) and in the book (Milanese et al., 1996). The paper is organized as follows. A brief review of background results on the relaxation of semialgebraic optimization problems through the theory of moments is presented in Section 2. Section 3 is devoted to the problem formulation. A detailed analysis of the geometrical structure of the set of all stable linear systems is presented in Section 4. On the basis of such an analysis, suitable convex relaxation techniques to solve the nonconvex optimization problems formulated in Section 3 are presented in Section 5. In Section 6, accuracy and convergency properties of the relaxed bounds computed in Section 5 are discussed. A simulated example is reported in Section 7. All proofs of properties and propositions presented in the paper can be found in the Appendix.

\section{Notation and background results on con- strained polynomial optimization}

In this section we briefly review some preliminary results on the relaxation of semialgebraic optimization problems through the theory of moments. The interested reader is referred to (Lasserre, 2001) for further technical details.

\subsection{Polynomial representation and theory of moments}

Let us denote with $\mathcal{P}_{m}^{n}[x]$ the space of real-valued polynomials of degree at most $m$ in the variable $x=\left[x_{1}, x_{2}, \ldots, x_{n}\right]^{\mathrm{T}}$ and let $h$ be the canonical basis of

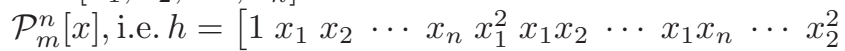

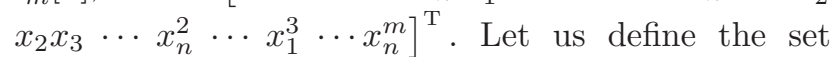
$\mathcal{A}_{m}=\left\{\alpha \in \mathbb{N}_{0}^{n}: \sum_{i=1}^{n} \alpha_{i} \leq m\right\}$, where $\alpha_{i}$ is the $i$-th component of the vector $\alpha$ and $\mathbb{N}_{0}^{n}$ denotes the set of $n$ dimensional nonnegative integer vectors. Then, the basis $h$ of the space $\mathcal{P}_{m}^{n}[x]$ can be written as $h=\left\{x^{\alpha}\right\}_{\alpha \in \mathcal{A}_{m}}$, where $x^{\alpha}=x_{1}^{\alpha_{1}} x_{2}^{\alpha_{2}} \cdots x_{n}^{\alpha_{n}}$.

Let $f$ and $g_{s}$ be in $\mathcal{P}_{m}^{n}[x]$. We denote the sequence $\mathbf{f}=\left\{f_{\alpha}\right\}_{\alpha \in \mathcal{A}_{m}}$ and $\mathbf{g}_{s}=\left\{g_{s \alpha}\right\}_{\alpha \in \mathcal{A}_{m}}$ as the coefficients of the polynomials $f$ and $g_{s}$, respectively, on the basis $h$, i.e. $f(x)=\sum_{\alpha \in A_{m}} f_{\alpha} x^{\alpha}$ and $g_{s}(x)=\sum_{\alpha \in A_{m}} g_{s \alpha} x^{\alpha}$. Let $p=\left\{p_{\alpha}\right\}_{\alpha \in \mathcal{A}_{m}}$ be the sequence of moments, up to order $m$, of a probability measure $\mu$ on $\mathbb{R}^{n}$, i.e. $p_{\alpha}=\int x^{\alpha} \mu(\mathrm{d} x)$. Let us denote with $M_{m}(p)$ the truncated moment matrix of order $m$ associated with the distribution $\mu$, i.e. $M_{m}(p)=\int h h^{\mathrm{T}} \mu(\mathrm{d} x)$ and $M_{m}\left(g_{k} p\right)$ the localizing matrix associated with the sequence of moments $p$ and with the polynomial $g_{k}(x)$.

\subsection{LMI-relaxation for polynomial problems}

Let us consider the constrained optimization problem

$$
f^{\star}=\min _{x \in \mathcal{S}} f(x)
$$

where $f \in \mathcal{P}_{m}^{n}[x]$ and $\mathcal{S} \subseteq \mathbb{R}^{n}$ is a compact semialgebraic set defined as $\mathcal{S}=\left\{x \in \mathbb{R}^{n}: g_{s}(x) \geq 0, s=1, \ldots, \Xi\right\}$, where $g_{s}$ is a real-valued polynomial in the variable $x \in$ $\mathbb{R}^{n}$ of degree $d_{s}=\operatorname{deg}\left(g_{s}\right)$, i.e. $g_{s} \in \mathcal{P}_{d_{s}}^{n}[x]$.

Let $\delta \in \mathbb{N}$ be such that $2 \delta \geq \max \left\{m, \max _{s} d_{s}\right\}$ and $h_{2 \delta}=$ $\left\{x^{\alpha}\right\}_{\alpha \in \mathcal{A}_{2 \delta}}$ be the canonical basis of the space $\mathcal{P}_{2 \delta}^{n}[x]$. Indeed, $f$ and $g$ belong to $\mathcal{P}_{2 \delta}^{n}[x]$. For any $\delta$ such that $2 \delta \geq \max \left\{m, \max _{s} d_{s}\right\}$, let us consider the semidefinite programming (SDP) problem

$$
\begin{aligned}
& f^{\delta}=\min _{p} \sum_{\alpha \in \mathcal{A}_{2 \delta}} f_{\alpha} p_{\alpha} \\
& \text { s.t. } M_{\delta}(p) \succeq 0, M_{\delta-\tilde{d}_{s}}\left(g_{s} p\right) \succeq 0, \quad s=1, \ldots, \Xi
\end{aligned}
$$

where $\tilde{d}_{s}=\left\lceil\frac{d_{s}}{2}\right\rceil, p=\left\{p_{\alpha}\right\}_{\alpha \in \mathcal{A}_{2 \delta}}$ is the sequence of moments up to order $2 \delta$ of some probability measure $\mu$ with support on $\mathcal{S}$, while $M_{\delta}(p)$ and $M_{\delta-\tilde{d}_{s}}\left(g_{s} p\right)$ are the moment matrix and the localizing matrix, respectively, associated with the moments $p$. Problem (2) is referred to as an LMI-relaxed problem of order $\delta$ of the polynomial problem (1). According to (Lasserre, 2001), the following results hold.

Theorem 1 If the functional $f(x)$ in (1) is linear and the feasible region $\mathcal{S}$ is defined by convex quadratic constraints, then $f^{\delta}=f^{\star}$ for all relaxation order $\delta \geq 1$.

Theorem 2 For all $\delta$ such that $2 \delta \geq \max \left\{m, \max _{s} d_{s}\right\}$, the following condition holds: $f^{\delta} \leq f^{\delta+1} \leq f^{\star}$.

Theorem 3 If there exists a real-valued polynomial $l(x): \mathbb{R}^{n} \rightarrow \mathbb{R}$ such that $\left\{x \in \mathbb{R}^{n}: l(x) \geq 0\right\}$ is a compact set, and $l(x)$ can be written as $l(x)=$ $l_{0}(x)+\sum_{s=1}^{\Xi} g_{s}(x) l_{s}(x) \forall x \in \mathbb{R}^{n}$, for some sums of squares polynomials $l_{s}(x)$, with $s=0, \ldots, \Xi$, then $\lim _{\delta \rightarrow \infty} f^{\delta}=f^{\star}$.

Although the convergence property in Theorem 3 is guaranteed as the relaxation order goes to infinity, exact global optimum $f^{\star}$ can be obtained in practice with a reasonably low relaxation order (see (Henrion and Lasserre, 2004) for a collection of test problems solved with relaxation order less or equal to 4 ). 


\section{Problem formulation}

Consider a SISO linear-time-invariant stable system mapping the noise-free input $u_{t}$ into the noise-free output $w_{t}$ according to the difference equation

$$
A\left(q^{-1}\right) w_{t}=B\left(q^{-1}\right) u_{t}
$$

where $A(\cdot)$ and $B(\cdot)$ are polynomials in the backward shift operator $q^{-1}\left(q^{-1} w_{t}=w_{t-1}\right)$ of the form $A\left(q^{-1}\right)=$ $1+a_{1} q^{-1}+\ldots+a_{n a} q^{-n a}$ and $B\left(q^{-1}\right)=b_{0}+b_{1} q^{-1}+$ $\ldots+b_{n b} q^{-n b}$. Let $r_{t}$ and $y_{t}$ be the noise-corrupted measurements of the input and output signals respectively:

$$
r_{t}=u_{t}+\xi_{t}, \quad y_{t}=w_{t}+\eta_{t},
$$

where $\xi_{t}$ and $\eta_{t}$ are measurement uncertainties which are assumed to range within given bounds $\Delta \xi_{t}$ and $\Delta \eta_{t}$ respectively, that is:

$$
\left|\xi_{t}\right| \leq \Delta \xi_{t}, \quad\left|\eta_{t}\right| \leq \Delta \eta_{t}
$$

Let $\theta \in \mathbb{R}^{\gamma}$, with $\gamma=n a+n b+1$, be the collection of the unknown parameters to be estimated, i.e. $\theta^{\mathrm{T}}=\left[\begin{array}{lllllll}a_{1} & \ldots & a_{n a} & b_{0} & b_{1} & \ldots & b_{n b}\end{array}\right]$. Given $N$ samples of the signals $r_{t}$ and $y_{t}$, the set $\mathcal{D}_{\theta}$ of all parameters $\theta$ consistent with measurements, error bounds and the assumed model structure is described by $(3)-(5)$, that is

$$
\begin{gathered}
\mathcal{D}_{\theta}=\left\{\theta \in \mathbb{R}^{\gamma}: A\left(q^{-1}\right)\left(y_{t}-\eta_{t}\right)=B\left(q^{-1}\right)\left(r_{t}-\xi_{t}\right),\right. \\
\left.\left|\xi_{t}\right| \leq \Delta \xi_{t}, \quad\left|\eta_{t}\right| \leq \Delta \eta_{t} ; t=1, \ldots, N\right\} .
\end{gathered}
$$

The exact feasible parameter set $\mathcal{D}_{\theta}$ is a nonconvex set described by nonlinear inequalities. As a consequence, parameters bounds could not be easily computed on the basis of $\mathcal{D}_{\theta}$. In order to overcome this problem, convex outer approximation $\mathcal{D}_{\theta}^{\prime}$ of $\mathcal{D}_{\theta}$, i.e. $\mathcal{D}_{\theta}^{\prime} \supseteq \mathcal{D}_{\theta}$, has been proposed in literature, see e.g. (Cerone, 1993a; Cerone, 1993b; Cerone et al., 2011; Cerone et al., 2010), to efficiently compute guaranteed bounds on each parameter $\theta_{j}$ by solving the optimization problems

$$
\underline{\theta}_{j}=\min _{\theta \in \mathcal{D}_{\theta}^{\prime}} \theta_{j}, \quad \bar{\theta}_{j}=\max _{\theta \in \mathcal{D}_{\theta}^{\prime}} \theta_{j} .
$$

In this work we will consider the description of the set $\mathcal{D}_{\theta}^{\prime}$ presented in (Cerone, 1993a), where $\mathcal{D}_{\theta}^{\prime}$ is described as a union of at most $2^{\gamma}$ convex regions, i.e. $\mathcal{D}_{\theta}^{\prime}=\bigcup_{i=1}^{2^{\gamma}} \mathcal{D}_{\theta i}^{\prime}$, where each set $\mathcal{D}_{\theta i}^{\prime}$ is a polytope defined by $2 N+\gamma$ linear constraints in the variables $\theta$, i.e.

$$
\mathcal{D}_{\theta i}^{\prime}=\left\{\theta \in \mathbb{R}^{\gamma}: g_{i t}^{\prime}(\theta) \geq 0, t=1, \ldots, 2 N+\gamma\right\},
$$

where $g_{i t}^{\prime}(\theta) \geq 0$ is the generic linear constraint defining $\mathcal{D}_{\theta i}^{\prime}$ (see (Cerone, 1993a) for details). Therefore, param- eters bounds $\underline{\theta}_{j}$ and $\bar{\theta}_{j}$ can be computed as

$$
\underline{\theta}_{j}=\min _{i=1, \ldots, 2^{\gamma}} \underline{\theta}_{j i}, \quad \bar{\theta}_{j}=\max _{i=1, \ldots, 2^{\gamma}} \bar{\theta}_{j i}
$$

where

$$
\underline{\theta}_{j i}=\min _{\theta \in \mathcal{D}_{\theta i}^{\prime}} \theta_{j}, \quad \bar{\theta}_{j i}=\max _{\theta \in \mathcal{D}_{\theta i}^{\prime}} \theta_{j} .
$$

In order to guarantee well-posedness of problems (6), we assume that $\mathcal{D}_{\theta}^{\prime}$ is a bounded set, i.e. there exists a constant $R>0$ arbitrarily large such that $\|\theta\|_{\infty} \leq$ $R, \forall \theta \in \mathcal{D}_{\theta}^{\prime}$. In view of this assumption, all $\theta \in \mathcal{D}_{\theta}^{\prime}$ satisfy the inequality $\|\theta\|_{2}^{2} \leq \gamma R^{2}$. Thus, an equivalent description of $\mathcal{D}_{\theta i}^{\prime}$ can be given adding to the description of (7) the redundant constraint $\|\theta\|_{2}^{2} \leq \gamma R^{2}$, that is

$$
\begin{gathered}
\mathcal{D}_{\theta i}^{\prime}=\left\{\theta \in \mathbb{R}^{\gamma}: g_{i t}^{\prime}(\theta) \geq 0, t=1, \ldots, 2 N+\gamma\right. \\
\left.g_{i k}^{\prime}(\theta)=\gamma R^{2}-\|\theta\|_{2}^{2} \geq 0, k=2 N+\gamma+1\right\} .
\end{gathered}
$$

The alternative description of the set $\mathcal{D}_{\theta i}^{\prime}$ given by (10) will play a crucial role in proving a key property of the proposed identification method (see Property 3 later). Since the linear system is a-priori known to be stable, we constrain the parameters $\theta$ to belong to the set $\mathcal{A}_{\theta}^{S T}$, where $\mathcal{A}_{\theta}^{S T}$ is the set of all parameters $\theta$ such that the system modeled by (3) is BIBO stable, that is:

$$
\mathcal{A}_{\theta}^{S T}=\left\{\theta \in \mathbb{R}^{\gamma}: A(z, \theta) \neq 0 \forall z \in \mathcal{C},|z| \geq 1\right\}
$$

with $A(z, \theta)=z^{n a}+a_{1} z^{n a-1}+\ldots+a_{n a}$.

In order to explicitly take into account information on system stability, the set $\mathcal{D}_{\theta}^{*}$ of all parameters belonging to $\mathcal{D}_{\theta}^{\prime}$ and guaranteeing BIBO stability of the identified system will be considered, which is defined as:

$$
\mathcal{D}_{\theta}^{*}=\mathcal{D}_{\theta}^{\prime} \cap \mathcal{A}_{\theta}^{S T} .
$$

Then, parameters bounds $\underline{\theta}_{j}^{*}$ and $\bar{\theta}_{j}^{*}$ for stable system are

$$
\underline{\theta}_{j}^{*}=\min _{\theta \in \mathcal{D}_{\theta}^{*}} \theta_{j}, \quad \bar{\theta}_{j}^{*}=\max _{\theta \in \mathcal{D}_{\theta}^{*}} \theta_{j},
$$

and the parameter uncertainty intervals for stable linear systems are defined as $P U I_{j}^{*}=\left[\underline{\theta}_{j}^{*}, \bar{\theta}_{j}^{*}\right]$, while the parameter uncertainty intervals computed without taking into account stability constraints are $P U I_{j}=\left[\underline{\theta}_{j} ; \bar{\theta}_{j}\right]$.

Remark 1 In this section we have formulated the problem of enforcing stability constraints in set-membership identification with reference to the general case where both input and output signals are corrupted by bounded noise (EIV setup). Due to the inherent complex structure of the exact feasible set $\mathcal{D}_{\theta}$ for the EIV case, the convex outer approximation $\mathcal{D}_{\theta}^{\prime}$ of $\mathcal{D}_{\theta}$ is considered, leading to the set $\mathcal{D}_{\theta}^{*}$ which is an outer approximation of the set $\mathcal{D}_{\theta} \cap \mathcal{A}_{\theta}^{S T}$ of all parameters belonging to $\mathcal{D}_{\theta}$ and 
guaranteeing BIBO stability of the identified system. It is worth noting that the conservativeness of the outer approximation $\mathcal{D}_{\theta}^{*}$ depends only on the choice of considering the general EIV setup, while the constraints arising from the a-priori information on the system stability are exactly taken into account in the definition of the set $\mathcal{A}_{\theta}^{S T}$. For example, $\mathcal{D}_{\theta}^{*}$ is an exact description of the set of all parameters belonging to $\mathcal{D}_{\theta}$ and guaranteeing BIBO stability of the identified system when the equation error framework is considered, which corresponds to the case $\xi_{t}=0$ and $\eta_{t}=\frac{1}{A\left(q^{-1}\right)} \eta_{t}^{\prime}$, with $\eta_{t}^{\prime}$ bounded

In the next section a detailed analysis of the geometrical structure of the set $\mathcal{A}_{\theta}^{S T}$ is presented. On the basis of such an analysis, guaranteed parameter uncertainty intervals are computed in Section 5.

\section{Analysis of the mathematical structure of the set $\mathcal{A}_{\theta}^{S T}$}

A necessary and sufficient condition for BIBO stability of the discrete time linear system described by $(3)$ is that the coefficients $a_{1}, \ldots, a_{n a}$ of polynomial $A\left(q^{-1}\right)$ satisfy the Jury's test (Jury, 1964).

Jury's test (Jury, 1964)

The roots of the polynomial $A\left(q^{-1}\right)$ belong to the open unit circle if and only if:

$$
\begin{gathered}
A(1)>0, \quad(-1)^{n a} A(-1)>0, \quad\left|a_{n a}\right|<1, \\
\left|c_{n a-1}\right|<\left|c_{0}\right|, \quad\left|d_{n a-2}\right|<\left|d_{0}\right|, \ldots,\left|q_{2}\right|<\left|q_{0}\right|,
\end{gathered}
$$

where $c_{0}, d_{0}, \ldots, q_{0}, \ldots, c_{n a-1}, d_{n a-2}, \ldots, q_{2}, q_{0}$ are elements of the Jury's array reported in Table 1, i.e. $c_{n a-j_{c}}=\left|\begin{array}{cc}a_{n a} & a_{n a-j_{c}} \\ 1 & a_{j_{c}}\end{array}\right|, d_{n a-j_{d}}=\left|\begin{array}{cc}c_{n a-1} & c_{n a-j_{d}} \\ c_{0} & c_{j_{d}}\end{array}\right|$, with $a_{0}=1$ and $|\cdot|$ denoting the determinant of a matrix.

Therefore, on the basis of Jury's criterion, the set $\mathcal{A}_{\theta}^{S T}$ defined in (11) can be described as the set of all the parameters $\theta$ which satisfy inequalities (14)-(15).

Topological features of the set $\mathcal{A}_{\theta}^{S T}$ are highlighted by the following property.

Table 1

Jury's array.

\begin{tabular}{ccclccc}
\hline$a_{n a}$ & $a_{n a-1}$ & $a_{n a-2}$ & $\ldots$ & $a_{2}$ & $a_{1}$ & 1 \\
1 & $a_{1}$ & $a_{2}$ & $\ldots$ & $a_{n a-2}$ & $a_{n a-1}$ & $a_{n a}$ \\
$c_{n a-1}$ & $c_{n a-2}$ & $c_{n a-3}$ & $\ldots$ & $c_{1}$ & $c_{0}$ & \\
$c_{0}$ & $c_{1}$ & $c_{2}$ & $\ldots$ & $c_{n a-2}$ & $c_{n a-1}$ & \\
$d_{n a-2}$ & $d_{n a-3}$ & $d_{n a-4}$ & $\ldots$ & $d_{0}$ & & \\
$\vdots$ & $\vdots$ & $\vdots$ & $\vdots$ & & & \\
$q_{2}$ & $q_{1}$ & $q_{0}$ & & & & \\
\hline
\end{tabular}

Property 1 If $n a \geq 2, \mathcal{A}_{\theta}^{S T}$ is the union of $2^{n a-2}$ semialgebraic sets, that is

$$
\mathcal{A}_{\theta}^{S T}=\bigcup_{k=1}^{2^{n a-2}} \mathcal{A}_{\theta k}^{S T}
$$

where $\mathcal{A}_{\theta k}^{S T}$ is a semialgebraic set in $\mathbb{R}^{\gamma}$ defined by 4 linear inequalities and $3(n a-2)$ polynomial inequalities of degree at most $2^{n a-2}$.

In the following, we consider the compact description $\mathcal{A}_{\theta k}^{S T}=\left\{\theta \in \mathbb{R}^{\gamma}: g_{k s}^{S T}(\theta) \geq 0, s=1, \ldots, 4+3(n a-2)\right\}$, where the symbols $g_{k s}^{S T}(\theta) \geq 0, s=1, \ldots, 4+3(n a-2)$ are used to describe the $4+3(n a-2)$ constraints defining $\mathcal{A}_{\theta k}^{S T}$ according to Property 1.

Remark 2 Inequalities (15) can be written as

$$
c_{n a-1}^{2}<c_{0}^{2}, \quad d_{n a-2}^{2}<d_{0}^{2}, \quad \ldots, \quad q_{2}^{2}<q_{0}^{2} .
$$

Therefore, the set $\mathcal{A}_{\theta}^{S T}$ can be also written as a single semialgebraic set defined by the polynomial inequalities (14) and (17) in the system parameters $a_{1}, \ldots, a_{n a}$. In such a description of $\mathcal{A}_{\theta}^{S T}$, the maximum degree of the polynomial constraints defining $\mathcal{A}_{\theta}^{S T}$ is $2^{n a-1}$, given by the degree of the polynomials $q_{0}^{2}$ and $q_{2}^{2}$.

\section{Computation of $P U I^{*} s$ by means of LMI re- laxation techniques}

In this section a procedure which numerically solves identification problems (13) is discussed.

Thanks to Property 1 , the set $\mathcal{D}_{\theta}^{*}$ in $(12)$ is the union of $2^{\gamma+n a-2}=2^{2 n a+n b-1}$ semialgebraic sets $\mathcal{D}_{\theta i k}^{*}$, that is:

$$
\mathcal{D}_{\theta}^{*}=\bigcup_{i=1}^{2^{\gamma}} \bigcup_{k=1}^{2^{n a-2}} \mathcal{D}_{\theta i k}^{*}
$$

where

$$
\mathcal{D}_{\theta i k}^{*}=\mathcal{D}_{\theta i}^{\prime} \cap \mathcal{A}_{\theta k}^{S T}
$$

Therefore, bounds $\underline{\theta}_{j}^{*}$ and $\bar{\theta}_{j}^{*}$ in (13) can be obtained by solving the optimization problems

$$
\underline{\theta}_{j}^{*}=\min _{\substack{i=1, \ldots, 2^{\gamma} \\ k=1, \ldots, 2^{\text {na-2 }}}} \underline{\theta}_{j i k}^{*}, \bar{\theta}_{j}^{*}=\max _{\substack{i=1, \ldots, 2^{\gamma} \\ k=1, \ldots, 2^{\text {na-2 }}}} \bar{\theta}_{j i k}^{*}
$$

where

$$
\underline{\theta}_{j i k}^{*}=\min _{\theta \in \mathcal{D}_{\theta i k}^{*}} \theta_{j}, \quad \bar{\theta}_{j i k}^{*}=\max _{\theta \in \mathcal{D}_{\theta i k}^{*}} \theta_{j} .
$$

Remark 3 Since the constraints described in (14)-(15) are strict inequalities, the feasible region $\mathcal{D}_{\theta i k}^{*}$ is not 
guaranteed to be a closed set. As a consequence, solutions to optimization problems (21) are not guaranteed to exist. A possible way to overcome such a technical problem is to modify constraints (14)-(15) as $A(1) \geq$ $\epsilon,(-1)^{n a} A(-1) \geq \epsilon,\left|a_{n a}\right| \leq 1-\epsilon,\left|c_{n a-1}\right| \leq\left|c_{0}\right|-$ $\epsilon,\left|d_{n a-2}\right| \leq\left|d_{0}\right|-\epsilon, \ldots,\left|q_{2}\right| \leq\left|q_{0}\right|-\epsilon$, where $\epsilon>0$ can be chosen arbitrarily small.

Polynomial optimization problems (21) are relaxed by applying the theory of moment method presented in Section 2.2 for a relaxation order $\delta \geq \underline{\delta}=\left\lceil\frac{2^{n a-2}}{2}\right\rceil$. This leads to the SDP problems:

$$
\underline{\theta}_{j i k}^{* \delta}=\min _{p \in \mathcal{D}_{\theta i k}^{* \delta}} \sum_{\alpha \in \mathcal{A}_{2 \delta}} f_{\alpha}^{j} p_{\alpha}, \quad \bar{\theta}_{j i k}^{* \delta}=\max _{p \in \mathcal{D}_{\theta i k}^{* \delta}} \sum_{\alpha \in \mathcal{A}_{2 \delta}} f_{\alpha}^{j} p_{\alpha} .
$$

More precisely, the feasible region $\mathcal{D}_{\theta i k}^{* \delta}$ for problems (22) is an LMI set defined as

$$
\begin{aligned}
\mathcal{D}_{\theta i k}^{* \delta}= & \left\{p: M_{\delta}(p) \succeq 0, M_{\delta}\left(g_{i t}^{\prime} p\right) \succeq 0, M_{\delta}\left(g_{k s}^{S T} p\right) \succeq 0,\right. \\
& t=1, \ldots, 2 N+\gamma+1, s=1, \ldots, 4+3(n a-2)\}
\end{aligned}
$$

where $M_{\delta}(p)$ is the truncated moment matrix of order $\delta$ associated to the moment sequence $p=\left\{p_{\alpha}\right\}_{\alpha \in \mathcal{A}_{2 \delta}}$, while $M_{\delta}\left(g_{i t}^{\prime} p\right)$ and $M_{\delta}\left(g_{k s}^{S T} p\right)$ are the localizing matrices associated with the constraints $g_{i t}^{\prime}(\theta) \geq 0$ and $g_{k s}^{S T}(\theta) \geq$ 0 respectively. The terms $f_{\alpha}^{j}$ are the elements of the sequence $\mathbf{f}^{j}=\left\{f_{\alpha}^{j}\right\}_{\alpha \in \mathcal{A}_{2 \delta}}$, which is the sequence of the coefficients of the functional $\theta_{j}$ in the basis $\left\{\theta^{\alpha}\right\}_{\alpha \in \mathcal{A}_{2 \delta} \text {. }}$.

For a given relaxation order $\delta \geq \underline{\delta}$, let us define the $\delta$ relaxed bounds $\underline{\theta}_{j}^{* \delta}$ and $\bar{\theta}_{j}^{* \delta}$ for the parameter $\theta_{j}$ as

$$
\underline{\theta}_{j}^{* \delta}=\min _{\substack{i=1, \ldots, 2^{\gamma} \\ k=1, \ldots, 2^{\text {na-2 }}}} \underline{\theta}_{j i k}^{* \delta}, \bar{\theta}_{j}^{* \delta}=\max _{\substack{i=1, \ldots, 2^{\gamma} \\ k=1, \ldots, 2^{\text {na-2 }}}} \bar{\theta}_{j i k}^{* \delta}
$$

Then, for each $j=1, \ldots, \gamma$, the $\delta$-relaxed parameter uncertainty intervals are defined as $P U I_{j}^{* \delta}=\left[\underline{\theta}_{j}^{* \delta}, \bar{\theta}_{j}^{* \delta}\right]$. The following proposition provides a sufficient condition to check if the optimal solution $\underline{\theta}_{j i k}^{*}$ to the minimization problem in (21) is achieved by the LMI-relaxed minimization problem (22) for a finite value of $\delta$.

Proposition 1 (Global optimality test)

Let $\tilde{p}=\left\{\tilde{p}_{\alpha}\right\}_{\alpha \in \mathcal{A}_{2 \delta}}$ be a global minimizer of (22) for $\delta \geq \underline{\delta}$. If $\tilde{\theta}=\left[\tilde{p}_{10 \cdots 0}, \tilde{p}_{01 \cdots 0}, \cdots \tilde{p}_{00 \cdots 1}\right]^{\mathrm{T}} \in \mathbb{R}^{\gamma}$ belongs to the FPS $D_{\theta}^{*}$, then

$$
\underline{\theta}_{j i k}^{*}=\underline{\theta}_{j i k}^{* \delta}
$$

Proposition 1 provides a simple procedure to test a posteriori the global optimality of the computed solution by checking if $\tilde{\theta}$ satisfies the inequalities defining $\mathcal{D}_{\theta}^{*}$ in (12). Indeed, the same considerations in Proposition 1 hold in order to check if the optimal solution $\overline{\theta_{j i k}^{*}}$ to the maximization problem in (21) is achieved by the relaxedmaximization problem in (22) for a finite value of $\delta$.

Remark 4 The number of optimization problems to be solved when stability constraints are enforced increases by a factor of $2^{n a-2}$ since the set $\mathcal{A}_{\theta}^{S T}$ is split into $2^{n a-2}$ semialgebraic sets. Besides, if the description of $\mathcal{A}_{\theta}^{S T}$ proposed by Remark 2 is used instead of the description of $\mathcal{A}_{\theta}^{S T}$ in Property 1, enforcing stability constraints does not increase the number of problems to be solved. However, in such a case, the set $\mathcal{A}_{\theta}^{S T}$ is described by $2 \cdot 2^{n a-2}$-degree polynomial inequalities, differently from the $2^{n a-2}$-degree polynomial constraints defining $\mathcal{A}_{\theta k}^{S T}$. As a consequence, the computational complexity of the corresponding SDP-problem that relaxes the identification problem is higher with respect to the computational complexity of the SDP-relaxed problems (22) which are obtained on the basis of the description of $\mathcal{A}_{\theta}^{S T}$ given by Property 1.

\section{Properties of relaxed parameter uncertainty intervals $P U I_{j}^{* \delta}$}

The following properties highlight some interesting features of the intervals $P U I_{j}^{* \delta}$.

\section{Property 2 Guaranteed relaxed uncertainty in-} tervals $P U I_{j}^{* \delta}$

For all $\delta \geq \underline{\delta}$, the true unknown parameter $\theta_{j}$ is guaranteed to belong to the $\delta$-relaxed parameter uncertainty interval $P U I_{j}^{* \delta}$, that is $\theta_{j} \in P U I_{j}^{* \delta}$.

Property 3 Monotone convergence to tight parameter uncertainty interval $P U I_{j}^{*}$

For all $\delta \geq \underline{\delta}$, the $\delta$-relaxed parameter uncertainty interval $P U I_{j}^{* \delta}$ becomes tighter as $\delta$ increases, that is

$$
\underline{\theta}_{j}^{* \delta} \leq \underline{\theta}_{j}^{* \delta+1}, \quad \bar{\theta}_{j}^{* \delta} \geq \bar{\theta}_{j}^{* \delta+1}
$$

Furthermore, the $\delta$-relaxed parameter uncertainty interval $P U I_{j}^{* \delta}$ converges to the tight interval $P U I_{j}^{*}$ as the relaxation order $\delta$ goes to infinity, i.e.

$$
\lim _{\delta \rightarrow \infty} \underline{\theta}_{j}^{* \delta}=\underline{\theta}_{j}^{*}, \quad \lim _{\delta \rightarrow \infty} \bar{\theta}_{j}^{* \delta}=\bar{\theta}_{j}^{*}
$$

Property 4 Accuracy improvement of $P U I_{j}^{* \delta}$ over $P U I_{j}$

For all $\delta \geq \underline{\delta}$, the $\delta$-relaxed stable parameter uncertainty interval $P U I_{j}^{* \delta}$ is tighter than the interval $P U I_{j}$, where no information on system stability is exploited, that is: $P U I_{j}^{* \delta} \subseteq P U I_{j}$, for all $j=1, \ldots, \gamma$. 


\section{A simulated example}

In this section a simulated example is presented in order to highlight the improvement obtained in the computation of the parameter bounds when information on stability is explicitly taken into account. A fourth order system is considered, with $\theta^{\mathrm{T}}=$ $\left[\begin{array}{llllllll}a_{1} & a_{2} & a_{3} & a_{4} & b_{1} & b_{2} & b_{3} & b_{4}\end{array}\right]=\left[\begin{array}{llllll}1.453 & 1.013 & 1.415 & 0.967\end{array}\right.$ $2.0551 .735-1.493-1.663]$. The length of the data sequence is $N=300$. The system is excited by a random input sequence $u_{t}$ uniformly distributed in $[-1,+1]$. Both input and output signals are corrupted by random additive noises $\xi_{t}$ and $\eta_{t}$, uniformly distributed in $\left[-\Delta \xi_{t},+\Delta \xi_{t}\right]$ and $\left[-\Delta \eta_{t},+\Delta \eta_{t}\right]$, respectively. The chosen error bounds $\Delta \xi_{t}$ and $\Delta \eta_{t}$ are such that the signal to noise ratios on the input and on the output are 31 $\mathrm{dB}$ and $30 \mathrm{~dB}$, respectively. First, bounds $\underline{\theta}_{j}$ and $\bar{\theta}_{j}$ are evaluated without enforcing stability constraints. Then, stability constraints are considered when computing bounds $\underline{\theta}_{j}^{*}$ and $\bar{\theta}_{j}^{*}$, solving problems $(22)$ with a relaxation order $\delta=2$. The freeware software GloptiPoly 3 (Henrion and Lasserre, 2003) is employed to convert polynomial problems (13) to the corresponding SDPrelaxed problems $(22)$, which are numerically solved by the solver SeDuMi. Table 2 shows the values of the central estimate $\theta_{j}^{c}=\frac{\bar{\theta}_{j}+\underline{\theta}_{j}}{2}$ and the uncertainty bounds $\Delta \theta_{j}=\frac{\bar{\theta}_{j}-\underline{\theta}_{j}}{2}$ obtained without imposing stability constraints and computed values of the central estimate $\theta_{j}^{c *}=\frac{\bar{\theta}_{j}^{*}+\underline{\theta}_{j}^{*}}{2}$ and the uncertainty bounds $\Delta \theta_{j}^{*}=\frac{\bar{\theta}_{j}^{*}-\underline{\theta}_{j}^{*}}{2}$ obtained when stability constraints are enforced. Results reported in Table 2 show that taking into account stability constraints leads to a significant reduction of parameters uncertainty for both the coefficients of the denominator $A\left(q^{-1}\right)$, and the coefficients of numerator $B\left(q^{-1}\right)$, although stability constraints involves polynomial $A\left(q^{-1}\right)$ only. The improvement on the estimation accuracy is relevant for the denominator parameters $a_{1}$, $a_{2}, a_{3}$ and $a_{4}$ as shown by the value of $\Delta \theta_{j}^{*}$ which, is at least $30 \%$ less than $\Delta \theta_{j}$, for $j=1,2,3,4$.

Numerical computation is carried out with Matlab 7.4 run on a $2.40-\mathrm{GHz}$ Intel Pentium IV with 3 GB RAM. The elapsed time to compute a single parameter bound $\underline{\theta}_{j}$ or $\bar{\theta}_{j}$ is between $0.5 \mathrm{~s}$ and $0.6 \mathrm{~s}$, while the time taken by the solver SeDuMi to compute a single parameter bound $\underline{\theta}_{j}^{*}$ or $\bar{\theta}_{j}^{*}$ is between $45 \mathrm{~s}$ and $52 \mathrm{~s}$. The reason of such an increasing in the computational time is due to the fact that SDP problems are solved when stability constraints are enforced, while LP optimization problems are solved to compute parameter bounds $\underline{\theta}_{j}$ and $\bar{\theta}_{j}$ without imposing stability constraints.

It is presently difficult to apply the discussed procedure to the identification of system of order higher than 4 because the memory required by the solver SeDuMi grows rapidly with the number of decision variables (for example, for a fifth order system, the memory required is roughly $16 \mathrm{~GB})$. However, the development of new algorithms to solve SDP problems is growing rapidly.
Table 2

Parameter central estimates $\left(\theta_{j}^{c}\right)$ and uncertainty bounds $\Delta \theta_{j}$ computed without stability constraints vs parameter central estimates $\left(\theta_{j}^{c *}\right)$ and parameter uncertainty bounds $\Delta \theta^{*}$ computed enforcing stability constraints.

\begin{tabular}{ccclll}
$\Delta \theta^{\circ}$ computed enforcing stability constraints. \\
\hline Parameter & True value & $\theta_{j}^{c}$ & $\Delta \theta_{j}$ & $\theta_{j}^{c *}$ & $\Delta \theta_{j}^{*}$ \\
$a_{1}$ & 1.453 & 1.547 & 0.528 & 1.409 & 0.258 \\
$a_{2}$ & 1.013 & 1.119 & 0.376 & 1.031 & 0.266 \\
$a_{3}$ & 1.415 & 1.470 & 0.408 & 1.370 & 0.270 \\
$a_{4}$ & 0.967 & 1.108 & 0.478 & 0.839 & 0.161 \\
$b_{1}$ & 2.055 & 2.165 & 1.019 & 2.201 & 0.968 \\
$b_{2}$ & 1.735 & 2.104 & 1.666 & 1.934 & 1.479 \\
$b_{3}$ & -1.493 & -1.657 & 1.378 & -1.546 & 1.265 \\
$b_{4}$ & -1.663 & -1.797 & 1.229 & -1.813 & 1.210 \\
\hline
\end{tabular}

For example, the interior point algorithm proposed in (Roh and Vandenberghe, 2006) seems to be a promising method to efficiently solve SDP-problems (22). Nevertheless, possible directions for further research are related to the development of efficient algorithms for SDP problems with a large number of decision variables.

\section{Conclusion}

Set-membership identification of linear systems a priori known to be stable is considered in the paper. First, it is shown that explicit enforcement of stability constraints in the evaluation of parameter bounds leads to nonconvex optimization problems. Then, on the basis of a detailed analysis of the geometrical structure of the stability constraints arising from the Jury's test, a convex relaxation technique is proposed to compute global optima of such problems. The computed relaxed bounds are shown to converge monotonically to the exact ones as the relaxation order goes to infinity. Furthermore, a procedure is presented to check, a posteriori, tightness of the bounds computed with a finite value of relaxation order. Accuracy improvement over the parameter bounds computed without stability constraints, irrespective of the value of the relaxation order, is proven. Effectiveness of the proposed technique is shown by means of a simulated example. Conservativeness of the computed parameter bounds depends on the choice of considering the EIV setup. On the other hand, polynomial constraints arising from enforcing system stability are efficiently handled through the discussed LMI-relaxation technique.

The presented identification approach can be applied also outside the set-membership framework. For instance, the computation of least square estimate constrained to the set of parameters satisfying the Jury's test requires the solution to a set of polynomial problems of the same kind of those considered in the paper.

\section{References}

Cerone, V. (1993a). Feasible parameter set for linear models with bounded errors in all variable. Automatica 29(6), 1551-1555. 
Cerone, V. (1993b). Parameter bounds for armax models from records with bounded errors in variables. Int. J. Control $\mathbf{5 7}(1)$, 225-235.

Cerone, V. D. Piga and D. Regruto (2010). Set-membership EIV identification through LMI relaxation techniques. In: Proc. of the American Control Conference 2010. pp. 2158-2163.

Cerone, V. D. Piga and D. Regruto (2011). Improved parameters bounds for set-membership EIV problems. Int. Journal of Adaptive Control and Signal Processing 25(3), 208-227.

Chui, N.L.C. and J.M. Maciejowski (1996). Realization of stable models with subspace methods. Automatica 32(11), 15871595 .

Gestel, T. Van, J.A.K. Suykens, P.Ṽan Dooren and B.D̃e Moor (2001). Identification of stable models in subspace identification by using regularization. IEEE Trans. Automatic Control 46(9), 1416-1420.

Henrion, D. and J. B. Lasserre (2003). Gloptipoly: Global optimization over polynomials with Matlab and SeDuMi. ACM Transactions Math. Soft 29, 165-194.

Henrion, D. and J. B. Lasserre (2004). Solving nonconvex optimization problems. IEEE Control Systems Magazine 24(3), 72-83.

Janakiraman, P.A. and A. Bhattacharyya (1999). Stable output error identification scheme using periodic excitation signals. In: Proc. of IEEE International Symposium on Circuits and Systems. Vol. 5. pp. 539-542.

Jury, E. (1964). The theory and application of Z-transform method. Wiley. New York.

Lacy, S.T. and D. Bernstein (2003). Subspace identification with guaranteed stability using constrained optimization. IEEE Trans. Automatic Control 48(7), 1259-1263.

Lasserre, J. B. (2001). Global optimization with polynomials and the problem of moments. SIAM Journal on Optimization 11, 796-817.

Milanese, M. and A. Vicino (1991). Optimal estimation theory for dynamic sistems with set membership uncertainty: an overview. Automatica 27(6), 997-1009.

Milanese, M. Norton, J. Piet-Lahanier, H. and Walter, E. Eds.) (1996). Bounding approaches to system identification. Plenum Press. New York.

Pillonetto, G. and G. DeNicolao (2010). A new kernelbased approach for linear system identification. Automatica 46(1), 81-93.

Regalia, P.A. and P. Stoica (1995). Stability of multivariable leastsquares models. IEEE Signal. Processing Letters 2(10), 195196.

Roh, T. and L. Vandenberghe (2006). Discrete transforms, semidefinite programming and sum-of-squares representations of nonnegative polynomials. SIAM Journal on Optimization 16(4), 939-964.

Söderström, T. and P. Stoica (1981). On the stability of dynamic models obtained by least-squares identification. IEEE Trans. Automatic Control 26(2), 575-577.

Tugnait, J.K. and C. Tontiruttananon (1998). Identification of linear systems via spectral analysis given time-domain data: Consistency, reduced-order approximation, and performance analysis. IEEE Trans. Automatic Control 43(10), 1354-1373.

Walter, E. and H. Piet-Lahanier (1990). Estimation of parameter bounds from bounded-error data: a survey. Mathematics and Computers in simulation 32, 449-468.

\section{A Proofs of properties and propositions re- ported in the paper}

Proof of Property 1 The set $\mathcal{A}_{\theta}^{S T}$, defined by inequalities $(14)-(15)$, can be written as

$$
\mathcal{A}_{\theta}^{S T}=\mathcal{A}_{1} \cap \underbrace{\mathcal{C} \cap \mathcal{D} \cap \ldots \cap \mathcal{Q}}_{\text {intersection of } n a-2 \text { sets }}
$$

where

$$
\begin{aligned}
\mathcal{A}_{1}=\left\{\theta \in \mathbb{R}^{\gamma}\right. & \left.: A(1)>0,(-1)^{n a} A(-1)>0,\left|a_{n a}\right|<1\right\}, \\
\mathcal{C} & =\left\{\theta \in \mathbb{R}^{\gamma}:\left|c_{n a-1}\right|<\left|c_{0}\right|\right\}, \\
\mathcal{D} & =\left\{\theta \in \mathbb{R}^{\gamma}:\left|d_{n a-2}\right|<\left|d_{0}\right|\right\}, \\
\vdots & \\
\mathcal{Q} & =\left\{\theta \in \mathbb{R}^{\gamma}:\left|q_{2}\right|<\left|q_{0}\right|\right\} .
\end{aligned}
$$

Besides, $\mathcal{C}=\mathcal{C}_{1} \cup \mathcal{C}_{2}, \mathcal{D}=\mathcal{D}_{1} \cup \mathcal{D}_{2}$ and so on, up to $\mathcal{Q}=\mathcal{Q}_{1} \cup \mathcal{Q}_{2}$, where

$$
\begin{gathered}
\mathcal{C}_{1}=\left\{\theta \in \mathbb{R}^{\gamma}: \quad c_{0} \geq 0,-c_{0} \leq c_{n a-1} \leq c_{0}\right\}, \\
\mathcal{C}_{2}=\left\{\theta \in \mathbb{R}^{\gamma}: \quad c_{0}<0, c_{0} \leq c_{n a-1} \leq-c_{0}\right\}, \\
\mathcal{D}_{1}=\left\{\theta \in \mathbb{R}^{\gamma}: \quad d_{0} \geq 0,-d_{0} \leq d_{n a-2} \leq d_{0}\right\} \\
\quad \vdots \\
\mathcal{Q}_{2}=\left\{\theta \in \mathbb{R}^{\gamma}: \quad q_{0}<0, q_{0} \leq q_{2} \leq-q_{0}\right\} .
\end{gathered}
$$

Thus, equation (A.1) can be rewritten as:

$$
\begin{aligned}
\mathcal{A}_{\theta}^{S T}= & \mathcal{A}_{1} \cap\left(\mathcal{C}_{1} \cup \mathcal{C}_{2}\right) \cap\left(\mathcal{D}_{1} \cup \mathcal{D}_{2}\right) \ldots \cap\left(\mathcal{Q}_{1} \cup \mathcal{Q}_{2}\right)= \\
= & \bigcup_{\substack{c=1,2 \\
d=1,2 \\
\vdots \\
q=1,2}}\left(\mathcal{A}_{1} \cap \mathcal{C}_{c} \cap \mathcal{D}_{d} \cap \ldots \cap \mathcal{Q}_{q}\right)= \\
= & \underbrace{\left(\mathcal{A}_{1} \cap \mathcal{C}_{1} \ldots \cap \mathcal{Q}_{1}\right)}_{\mathcal{A}_{\theta 1}^{S T}} \cup \underbrace{\left(\mathcal{A}_{1} \cap \mathcal{C}_{1} \ldots \cap \mathcal{Q}_{2}\right)}_{\mathcal{A}_{\theta 2}^{S T}} \\
& \ldots \cup \underbrace{\left(\mathcal{A}_{1} \cap \mathcal{C}_{2} \cap \ldots \cap \mathcal{Q}_{2}\right)}_{\mathcal{A}_{\theta 2^{n a-2}}^{S T}}=\bigcup_{k=1, \ldots, 2^{n a-2}} \mathcal{A}_{\theta k}^{S T} .
\end{aligned}
$$

Since all the entries of the Jury's array are polynomial functions of variables $a_{1}, \ldots, a_{n a}$, each set $\mathcal{C}_{c}, \mathcal{D}_{d}, \ldots, \mathcal{Q}_{q}$ (for $c=1,2 ; d=1,2 ; \ldots ; q=1,2$ ) is defined by 3 polynomial inequality constraints in the unknown parameters $a_{1}, \ldots, a_{n a}$. Therefore, the sets $\mathcal{C}_{c}, \mathcal{D}_{d}, \ldots, \mathcal{Q}_{q}$ are semialgebraic regions in the parameters space $\mathbb{R}^{\gamma}$. Further, $\mathcal{A}_{1}$ is defined by 4 linear inequality constraints. Since each set $\mathcal{A}_{\theta k}^{S T}$, for $k=1, \ldots, 2^{n a-2}$, is given by the intersection of the polytope $\mathcal{A}_{1}$ and the $n a-2$ semialgebraic sets $\mathcal{C}_{c}, \mathcal{D}_{d}, \ldots, \mathcal{Q}_{q}$, for all possible combinations of the indexes $(c=1,2 ; d=1,2 ; \ldots ; q=1,2)$, it follows that $\mathcal{A}_{\theta k}^{S T}$ is a semialgebraic set in $\mathbb{R}^{\gamma}$, defined by the 4 linear inequalities describing $\mathcal{A}_{1}$ and the $3(n a-2)$ polynomial constraints describing $\mathcal{C}_{c}, \mathcal{D}_{d}, \ldots, \mathcal{Q}_{q}$. Then, the maximum degree of the polynomial constraints defining $\mathcal{A}_{\theta k}^{S T}$ is $2^{n a-2}$, given by the degree of the polynomials $q_{0}$ and $q_{2}$, which are the last entries of the Jury's table and which appear in the definition of $\mathcal{Q}_{1}$ and $\mathcal{Q}_{2}$. 
Proof of Proposition 1 Since $\tilde{\theta} \in D_{\theta}^{*}$ by hypothesis, then

$$
\underline{\theta}_{j i k}^{*} \leq \sum_{\alpha \in \mathcal{A}_{2 \delta}} f_{\alpha}^{j} \tilde{\theta}^{\alpha}
$$

Besides, since $\tilde{p}$ is a global minimizer for (22), then

$$
\underline{\theta}_{j i k}^{* \delta}=\sum_{\alpha \in \mathcal{A}_{2 \delta}} f_{\alpha}^{j} \tilde{p}^{\alpha} \leq \underline{\theta}_{j i k}^{*}, \text { for all } \delta \geq \underline{\delta}
$$

The right side of condition (A.3) holds since $\underline{\theta}_{j i k}^{* \delta} \leq \underline{\theta}_{j i k}^{*}$ for all $\delta \geq \underline{\delta}$ from Property 4. Then, from (A.2) and (A.3), condition (25) follows.

Proof of Property 2 From direct application of Theorem 2 to semialgebraic problems (21) and the corresponding SDP-relaxed problems (22), the following conditions can be trivially proven: $\underline{\theta}_{j}^{* \delta} \leq \underline{\theta}_{j}^{*}$ and $\bar{\theta}_{j}^{* \delta} \geq \bar{\theta}_{j}^{*}$, that is $P U I_{j}^{*} \subseteq P U I_{j}^{* \delta}$. Therefore, since $\theta_{j} \in P U I_{j}^{*}$, then $\theta_{j} \in P U I_{j}^{* \delta}$ as stated in Property 2.

Proof of Property 3 From direct application of Theorem 2 to problems (21), it follows:

$$
\underline{\theta}_{j i k}^{* \delta} \leq \underline{\theta}_{j i k}^{* \delta+1}, \quad \bar{\theta}_{j i k}^{* \delta} \geq \bar{\theta}_{j i k}^{* \delta+1}
$$

Then, from equations (A.4) and the definition of $\underline{\theta}_{j}^{*}$ and $\overline{\theta_{j}^{*}}$ in (24), equations (26) follow. We are left to prove equations (27). To this aim, consider the realvalued polynomial $l(\theta): \mathbb{R}^{\gamma} \rightarrow \mathbb{R}$ defined as $l(\theta)=$ $l_{0}(\theta)+\sum_{t=1}^{2 N+\gamma+1} l_{t}(\theta) g_{i t}^{\prime}(\theta)+\sum_{s=1}^{4+3(n a-2)} l_{s}(\theta) g_{k s}^{S T}(\theta)$, with $l_{0}(\theta)=0 ; l_{t}(\theta)=0$ for $t=1, \ldots, 2 N+\gamma ; l_{t}(\theta)=1$ for $t=2 N+\gamma+1 ; l_{s}(\theta)=0$ for $s=1, \ldots, 4+$ $3(n a-2)$. Therefore, $l(\theta)=\gamma R^{2}-\|\theta\|_{2}^{2}$. Then, because $l_{0}, l_{t}$ and $l_{s}$ are sum-of-squares polynomials for all $t=1, \ldots, 2 N+\gamma+1 ; s=1, \ldots, 4+3(n a-2)$ and the set $\left\{\theta \in \mathbb{R}^{\gamma}: l(\theta)=\gamma R^{2}-\|\theta\|_{2}^{2} \geq 0\right\}$ is compact, from direct application of Theorem 3 to problems (21), the following conditions hold for all $i=1, \ldots, 2^{\gamma}$; $k=1, \ldots, 2^{n a-2}$ :

$$
\lim _{\delta \rightarrow \infty} \underline{\theta}_{j i k}^{* \delta}=\underline{\theta}_{j i k}^{*}, \quad \lim _{\delta \rightarrow \infty} \bar{\theta}_{j i k}^{* \delta}=\bar{\theta}_{j i k}^{*}
$$

Then, from equations (A.5) and the definition of $\underline{\theta}_{j}^{*}$ and $\bar{\theta}_{j}^{*}$ in $(24)$, equations (27) follow.

Proof of Property 4 For $\delta \geq \underline{\delta}$, let us define the sets

$$
\begin{aligned}
D_{\theta i}^{\prime \delta}= & \left\{p: M_{\delta}(p) \succeq 0, M_{\delta}\left(g_{i t}^{\prime} p\right) \succeq 0,\right. \\
& t=1, \ldots, 2 N+n a+1\}, \\
\mathcal{A}_{\theta k}^{S T \delta}= & \left\{p: M_{\delta}(p) \succeq 0, M_{\delta}\left(g_{k s}^{S T} p\right) \succeq 0,\right. \\
& s=1, \ldots, 4+3(n a-2)\} .
\end{aligned}
$$

Indeed, the set $\mathcal{D}_{\theta i k}^{* \delta}$ in $(23)$ can be rewritten as

$$
\mathcal{D}_{\theta i k}^{* \delta}=\mathcal{D}_{\theta i}^{\prime} \cap \cap \mathcal{A}_{\theta k}^{S T \delta}
$$

From (A.8) and the definition of $\underline{\theta}_{j i k}^{* \delta}$ in (22), the following condition holds for all $j=1, \ldots, \gamma ; i=1, \ldots, 2^{\gamma}$; $k=1, \ldots, 2^{n a-2}$ :

$$
\underline{\theta}_{j i k}^{* \delta}=\min _{p \in \mathcal{D}_{\theta i k}^{* \delta}} \sum_{\alpha \in \mathcal{A}_{2 \delta}} f_{\alpha}^{j} p_{\alpha} \geq \min _{y \in \mathcal{D}_{\theta i}^{\prime \delta}} \sum_{\alpha \in \mathcal{A}_{2 \delta}} f_{\alpha}^{j} p_{\alpha} ; \quad \forall \delta \geq \underline{\delta} .
$$

Furthermore, since $\mathcal{D}_{\theta i}^{\prime}$ in (10) is defined by linear and convex quadratic constraints and the functional for problems (6) is linear, from Theorem 1 we have, for all $j=1, \ldots, \gamma ; i=1, \ldots, 2^{\gamma}$ :

$$
\min _{p \in \mathcal{D}_{\theta i}^{\prime \delta}} \sum_{\alpha \in \mathcal{A}_{2 \delta}} f_{\alpha}^{j} p_{\alpha}=\min _{\theta \in \mathcal{D}_{\theta i}^{\prime}} \theta_{j}=\underline{\theta}_{j i} ; \quad \forall \delta \geq \underline{\delta} .
$$

From (A.9) and (A.10), $\underline{\theta}_{j i k}^{* \delta} \geq \theta_{j i}$ for all $\delta \geq \underline{\delta}$ and for all $j=1, \ldots, \gamma ; i=1, \ldots, 2^{\gamma} ; \bar{k}=1, \ldots, 2^{\text {na-2}}$. Then, from the definitions of $\underline{\theta}_{j}^{* \delta}$ in (24) and $\underline{\theta}_{j}$ in (8), it follows

$$
\underline{\theta}_{j}^{* \delta} \geq \underline{\theta}_{j} \text { for all } j=1, \ldots, \gamma, \delta \geq \underline{\delta} .
$$

From similar considerations, it follows:

$$
\bar{\theta}_{j}^{* \delta} \leq \bar{\theta}_{j} \text { for all } j=1, \ldots, \gamma ; \delta \geq \underline{\delta} .
$$

Then, from (A.11), (A.12) and the definition of $P U I_{j}^{* \delta}$ and $P U I_{j}, P U I_{j}^{* \delta} \subseteq P U I_{j}$ as stated in Property 4. 